\title{
The stability of the hydroxylated (0001) surface of alpha-Al2O3
}

\author{
Lodziana, Zbigniew; Nørskov, Jens Kehlet; Stoltze, Per
}

Published in:

Journal of Chemical Physics

Link to article, DOI:

$10.1063 / 1.1574798$

Publication date:

2003

Document Version

Publisher's PDF, also known as Version of record

Link back to DTU Orbit

Citation (APA):

Lodziana, Z., Nørskov, J. K., \& Stoltze, P. (2003). The stability of the hydroxylated (0001) surface of alphaAl2O3. Journal of Chemical Physics, 118(24), 11179-11188. https://doi.org/10.1063/1.1574798

\section{General rights}

Copyright and moral rights for the publications made accessible in the public portal are retained by the authors and/or other copyright owners and it is a condition of accessing publications that users recognise and abide by the legal requirements associated with these rights.

- Users may download and print one copy of any publication from the public portal for the purpose of private study or research.

- You may not further distribute the material or use it for any profit-making activity or commercial gain

- You may freely distribute the URL identifying the publication in the public portal

If you believe that this document breaches copyright please contact us providing details, and we will remove access to the work immediately and investigate your claim. 


\title{
The stability of the hydroxylated (0001) surface of $\alpha-\mathrm{Al}_{2} \mathrm{O}_{3}$
}

\author{
Z. Łodziana and J. K. Nørskov \\ Center for Atomic-scale Materials Physics, DTU, Building 307, DK-2800 Lyngby, Denmark \\ P. Stoltze \\ Department of Chemistry and Applied Engineering Science Aalborg University Esbjerg, Niels Bohr Vej 8, \\ DK-6700 Esbjerg, Denmark
}

(Received 6 January 2003; accepted 25 March 2003)

\begin{abstract}
Self-consistent density functional calculations of the hydroxylated (0001) corundum surfaces are presented. It is demonstrated that the hydroxylated surfaces are the most stable under most, but not all, conditions. Hydroxylation significantly lowers the surface free energy of $\alpha$-alumina. The stability of the hydrated surface resolves the discrepancies between the morphology of the $\alpha$-alumina (0001) surface observed under ultra-high vacuum, and at ambient conditions. A method for the calculation of the equilibrium surface stoichiometry is proposed. The proposed approach provides a valuable connection between theoretical calculations and experiments with metal oxides. (C) 2003 American Institute of Physics. [DOI: 10.1063/1.1574798]
\end{abstract}

\section{INTRODUCTION}

The properties of ceramic surfaces and the nature of the metal-ceramic interface have been the subject of growing interest in recent years. ${ }^{1}$ In particular, the role of alumina is important, since it is commonly used as a support material or as a catalyst in its own right. ${ }^{2,3}$ Of the aluminas, the (0001) surface of corundum has been the testing ground of most of the new ideas. ${ }^{3-6}$ Only recently, experimental and theoretical methods have established a relatively clear picture of this surface in ultra high vacuum. ${ }^{5,7-10}$ A full understanding of the environment dependent structure and reactivity of the (0001) $\alpha-\mathrm{Al}_{2} \mathrm{O}_{3}$ surface is hampered, however, by some discrepancies between the experimental observations ${ }^{7-9}$ and theoretical predictions. ${ }^{5,10}$ The discrepancies are believed to be related to the hydration of the surface. ${ }^{4}$

Several authors have pointed out that the surface structure of a dense $\alpha-\mathrm{Al}_{2} \mathrm{O}_{3}$ could be fairly different under ultrahigh vacuum (UHV) and ambient conditions. ${ }^{4,10-14}$ Ahn and Rablais ${ }^{7}$ determined the structure of the $(1 \times 1)$ surface terminated by Al under UHV. Barth and Reichling ${ }^{9}$ observed the reconstructed $(\sqrt{31} \times \sqrt{31})$ surface also in UHV conditions. Both experiments observed hydroxylation of the surface as the pressure of water was increased. On the other hand, Eng et al. ${ }^{4}$ have shown that the structure of the (0001) surface of dense $\alpha$-phase alumina exposed to water vapor is part way between that of stoichiometric $\alpha-\mathrm{Al}_{2} \mathrm{O}_{3}$ and $\gamma-\mathrm{Al}(\mathrm{OH})_{3}$ (gibbsite, bayerite), i.e., different to that observed under UHV. ${ }^{7-9}$

Hydroxylation is of special importance for alumina, as surfaces of all phases of alumina are almost always covered by water, either dissociated or molecular. ${ }^{6,11}$ McHale et al. ${ }^{11,12}$ showed that the amount of surface hydroxyl groups varies from $\sim 15 \mathrm{OH} / \mathrm{nm}^{2}$ at a surface area of $150 \mathrm{~m}^{2} / \mathrm{g}$ and $600 \mathrm{~K}$ to $\sim 3 \mathrm{OH} / \mathrm{nm}^{2}$ at $30 \mathrm{~m}^{2} / \mathrm{g}$ and $1400 \mathrm{~K}$ for the porous $\alpha$ phase. The composition and the relative stability of different hydrated surfaces is important, since the key property of aluminas - the porosity - is strongly influenced by the sur- face energy ${ }^{15}$ and the latter must depend on the degree of hydroxylation.

Only those selected examples show the need to determine the structure and composition of the surface as a function of temperature, pressure and environment. This requires determination of the free energy and a proper description of the adsorption/desorption processes. The latter is crucial in determining the equilibrium $\mathrm{OH}$ coverage at a given $(T, p)$.

Theoretical investigations have already tried to meet this challenge for alumina. Wang et al. ${ }^{10}$ estimated the free energy of the $\alpha-\mathrm{Al}_{2} \mathrm{O}_{3}$ surface with respect to the chemical potential of $\mathrm{O}_{2}$ and $\mathrm{H}_{2}$. They reported high stability of the metal-terminated surface at large values of the oxygen chemical potential. Additionally they showed that the fully hydroxylated surface is always more stable than the metal terminated one. Another theoretical study ${ }^{6}$ of the wateralumina interaction clarified the mechanism of $\mathrm{H}_{2} \mathrm{O}$ dissociation on the (0001) surface of corundum. There is, however, no understanding at present of the transition from the clean to the fully hydroxylated state of the surface.

In the present paper, we address the problem of the influence of hydroxylation on the composition and stability of the (0001) surface of corundum. To do this a new, general method for calculating surface phase diagrams is developed. Contrary to the standard approach, ${ }^{10}$ the possibility of a continuous variation of the surface composition, and entropic effect for the surface modes of hydroxyl groups, are included. Using this method in conjunction with extensive density functional calculations of both ground state energies and the vibrational properties, we determine the surface phase diagram for the (0001) surface of $\alpha-\mathrm{Al}_{2} \mathrm{O}_{3}$ in a humid atmosphere and show that hydroxylated surfaces, are generally favored at most ambient conditions.

The paper is presented as follows: In the next section the model of $\alpha-\mathrm{Al}_{2} \mathrm{O}_{3}$ and the details of the DFT calculations are presented. Section III reviews the thermodynamic approach pinpointing the important issues related to the sur- 
face. The extension of this method toward constructing the surface phase diagram is presented in Sec. IV. Section V contains the results of the present calculations.

\section{METHOD OF CALCULATION}

The calculations were performed using density functional theory (DFT) with the ionic cores represented by ultrasoft pseudopotentials. The self-consistent gradient corrected GGA-PW91 ${ }^{16}$ electron density was determined by the iterative diagonalization of the Kohn-Sham Hamiltonian. ${ }^{17}$ The Brillouin zone was sampled according to the Monkhorst-Pack scheme with a $k$-point spacing of $\sim 0.1 \AA^{-1}$ in reciprocal space.

$\alpha-\mathrm{Al}_{2} \mathrm{O}_{3}$ has a rhombohedral symmetry $(R \overline{3} c)$ and the primitive unit cell contains two formula units. In the first step of the calculations the internal degrees of freedom and the lattice parameters of the primitive unit cell were optimized within the DFT approach. The fully relaxed lattice parameters are $a=5.151 \AA, \alpha=55.279^{\circ}$. The atomic positions are $(x, x, x), x=0.352$ for aluminum and $(0.5-z, 0.25, z), z$ $=0.556$ for oxygen. These values compare well to the experimental ${ }^{18,19}$ lattice parameters $a=5.128 \AA, \alpha=55.28^{\circ}$ and the internal positions of oxygen $x=0.352$ and aluminum $z=0.556$. A slight overestimate of the unit cell volume is typical for DFT calculations with the GGA exchangecorrelation functional. The agreement with other calculations ${ }^{15}$ is very good.

The (0001) surface of corundum was constructed in the hexagonal setting and $15 \AA$ of vacuum was added. The most stable surface termination is a single $\mathrm{Al}$ layer. ${ }^{5,7,10}$ The possibility of spontaneous reconstruction was not taken into account in the present studies, which are focused on the effects related to the hydroxylation. The stoichiometry of the gibbsite surface phase $\left[\mathrm{Al}(\mathrm{OH})_{3}\right]$ is limited to the topmost surface layer only, and the underlying bulk possesses corundum structure.

Slabs consisting of 4 to 6 oxygen layers were studied. One side of the slab was frozen in the bulk configuration and the other surface was fully relaxed. Extensive tests show that this model accurately represents the (0001) surface of corundum. The surface relaxation and the surface energy ${ }^{19}$ agrees very well with those reported in the literature. ${ }^{6}$ Since we are interested in studies of water adsorption at various coverages a larger than primitive supercell has to be used. For that purpose a $(2 \times 2)$ supercell was constructed from the optimized $(1 \times 1)$ geometry and the structural optimization was repeated for this larger surface. The $k$-point sampling was reduced appropriately, which results in minor changes of the atomic positions at the relaxed surface. The majority of the present calculations were performed on large supercells containing more than 80 atoms. The top view of the relaxed 2 $\times 2$ supercell is presented in Fig. 1. The adsorption sites of the hydroxyl groups are marked and will be described in Sec. V.

\section{THERMODYNAMIC MODEL}

In this section the thermodynamical/statistical formalism to describe the surface at equilibrium ${ }^{10,20-24}$ is presented and

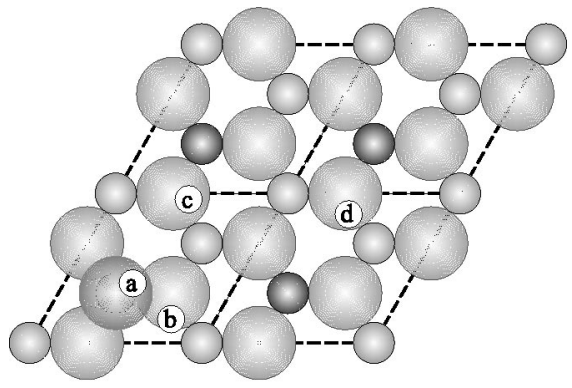

FIG. 1. Top view of the $(0001)$ surface of $\mathrm{Al}_{2} \mathrm{O}_{3}$ in the $(2 \times 2)$ supercell. Large circles represent oxygen atoms. Small gray circles represent aluminum (top Al is marked as darker). Small white circles with letters inside represent hydrogen. Hydrogen (a) atom with the darker oxygen atom beneath, are the $\mathrm{OH}$ groups on top of surface Al. $a, b, c, d$ denote the possible sites occupied by hydrogen after water dissociation. Only the topmost layer is shown for clarity.

an appropriate extension is formulated. The reader familiar with these methods may skip the introductory review.

The Gibbs free energy $G=H+p V-T S$ is the natural choice of the thermodynamic potential to describe thermodynamic properties of the system at atmospheric conditions $[(N, p, T)$ ensemble $]$.

To describe the equilibrium composition of the hydroxylated (0001) surface of $\alpha-\mathrm{Al}_{2} \mathrm{O}_{3}$ consider a partially hydroxylated slab of alumina consisting of $N_{\mathrm{Al}}$ aluminum atoms, $N_{\mathrm{O}}$ oxygen atoms, and $N_{\mathrm{H}}$ hydrogen atoms. For the formation of this slab from isolated atoms, we have the Haber cycle shown in Fig. 2 and consequently

$$
\Delta G_{1}+\Delta G_{2}+\Delta G_{3}+\Delta G_{4}=\Delta G_{5} .
$$

Below, each of the steps is considered individually.

Step 1: Is the formation of the $\mathrm{Al}_{2} \mathrm{O}_{3}$ crystal in the slab geometry. The formation energy of alumina equals

$$
\begin{aligned}
\Delta G_{1}= & N G_{\mathrm{Al}_{2} \mathrm{O}_{3}}^{0}-\left(2 N G_{\mathrm{Al}}^{0}+3 N G_{\mathrm{O}}^{0}\right) \\
& +N\left(\Delta H_{\mathrm{Al}_{2} \mathrm{O}_{3}}^{0}-T \Delta S_{\mathrm{Al}_{2} \mathrm{O}_{3}}\right) .
\end{aligned}
$$

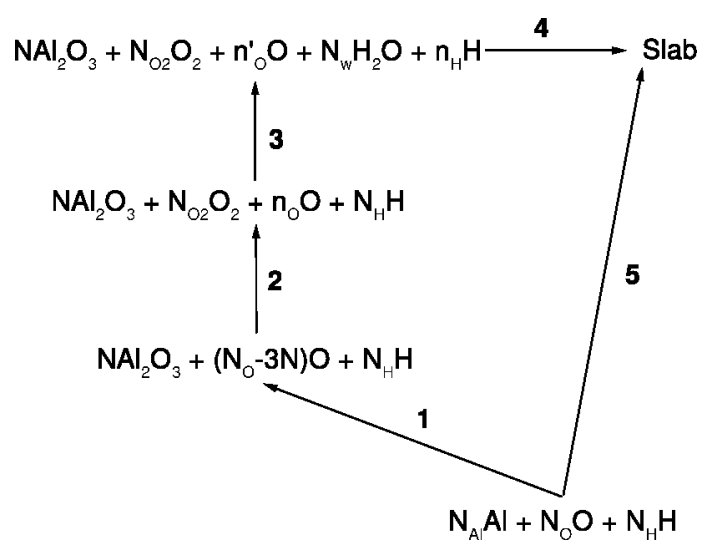

FIG. 2. The Haber cycle used in the formation of the thermodynamic model. Stoichiometric amounts of the elements are taken to form a slab of $N$ molecular units, $N_{\mathrm{O}_{2}}$ oxygen molecules $N_{\mathrm{H}_{2}}$ hydrogen molecules, and $N_{w}$ molecules of water. $n_{\mathrm{O}}=N_{\mathrm{O}}-3 N-2 N_{\mathrm{O}_{2}}, n^{\prime}{ }_{\mathrm{O}}=N_{\mathrm{O}}-3 N-2 N_{\mathrm{O}_{2}}-N_{w}, n_{\mathrm{H}}=N_{\mathrm{H}}$ $-2 N_{w}$. 
The superscripts indicate that the species are in the standard state $\left(T=298.15 \mathrm{~K}, p=p^{\ominus}=1 \mathrm{~atm}\right)$. The slab geometry adds the excess energy related to the presence of a free surface

$$
\Delta G_{\mathrm{ex}}=\Delta G_{1}-\Delta G_{1}^{\prime}=\Delta G_{1}-N\left(\Delta H_{\mathrm{Al}_{2} \mathrm{O}_{3}}-T \Delta S_{\mathrm{Al}_{2} \mathrm{O}_{3}}\right),
$$

where $G_{1}$ is the free energy of the slab and $G_{1}^{\prime}$ is the energy of the same amount of the bulk. When the slab is symmetric $G_{\text {ex }}$ equals the surface energy times the surface area. When the two sides of the slab are different $G_{\text {ex }}$ gives the separation energy (the sum of the surface energies of both sides of the slab).

Step 2: Is the formation of oxygen and hydrogen molecules in the gas phase

$$
\begin{aligned}
\Delta G_{2}= & N_{\mathrm{O}_{2}} \Delta G_{\mathrm{O}_{2}}+N_{\mathrm{H}_{2}} \Delta G_{\mathrm{H}_{2}} \\
= & N_{\mathrm{O}_{2}}\left(\Delta H_{\mathrm{O}_{2}}-T \Delta S_{\mathrm{O}_{2}}+R T \ln \left(\frac{p_{\mathrm{O}_{2}}}{p^{\ominus}}\right)\right) \\
& +N_{\mathrm{H}_{2}}\left(\Delta H_{\mathrm{H}_{2}}-T \Delta S_{\mathrm{H}_{2}}+R T \ln \left(\frac{p_{\mathrm{H}_{2}}}{p^{\ominus}}\right)\right) .
\end{aligned}
$$

This is a standard thermodynamic expression for the pressure/temperature dependence of the free energy. Details of the chemical potentials of gases and liquids are presented in the Appendix.

Step 3: Is the formation of water

$$
\begin{aligned}
\Delta G_{3} & =N_{w}\left(\Delta G_{\mathrm{O}}+2 \Delta G_{\mathrm{H}}\right) \\
& =N_{w}\left(\Delta H_{w}-T \Delta S_{w}+R T \ln \left(\frac{p_{W}}{p^{\ominus}}\right)\right) .
\end{aligned}
$$

This refers to water in the vapor state. In reality, the saturated vapor in equilibrium with the liquid shall be considered. In this case $p_{W}=\gamma p^{\text {sat }} \phi^{\text {sat }}$, where $\phi^{\text {sat }}$ is the fugacity of the saturated vapor, and $\gamma$ is the chemical activity of the liquid (see the Appendix).

Step 4: Is the formation of the hydroxylated/oxidized slab by adsorption of water, oxygen or hydrogen. Note that the system is in equilibrium with $\mathrm{Al}_{2} \mathrm{O}_{3}, \mathrm{H}_{2} \mathrm{O}(g), \mathrm{O}_{2}(g)$, and $\mathrm{H}_{2}(g)$ so that $\Delta G_{4}=0$.

Step 5: Is the formation of the system from isolated atoms

$$
\Delta G_{5}=N\left(\Delta H_{S}-T \Delta S_{S}\right)=G_{S}
$$

which expresses the free energy of the slab. Combining all the equations, the equilibrium reads

$$
\begin{aligned}
& N\left(G_{\mathrm{Al}_{2} \mathrm{O}_{3}}-T \Delta S_{\mathrm{Al}_{2} \mathrm{O}_{3}}\right)+N_{\mathrm{O}_{2}}\left(G_{\mathrm{O}_{2}}-T \Delta S_{\mathrm{O}_{2}}+R T \ln \left(\frac{p_{\mathrm{O}_{2}}}{p^{\ominus}}\right)\right) \\
& +N_{\mathrm{H}_{2}}\left(G_{\mathrm{H}_{2}}-T \Delta S_{\mathrm{H}_{2}}+R T \ln \left(\frac{p_{\mathrm{H}_{2}}}{p^{\ominus}}\right)\right) \\
& +N_{w}\left(G_{w}-T \Delta S_{w}+R T \ln \left(\frac{p_{W}}{p^{\ominus}}\right)\right)=G_{S}
\end{aligned}
$$

The Gibbs free energy of the pure elements can be expressed via the chemical potentials $\mu=(\partial G / \partial N)_{T, p}=(\partial F / \partial N)_{T, V}$, with the appropriate $(T, p)$ variation of $\mu$ taken into account. The steady state of the equilibrium imposes some constraints on the chemical potential: $\mu_{\mathrm{Al}_{2} \mathrm{O}_{3}}=2 \mu_{\mathrm{Al}}+3 \mu_{\mathrm{O}}^{\prime}, \quad \mu_{\mathrm{H}_{2} \mathrm{O}}$ $=\mu_{\mathrm{H}_{2}}+\frac{1}{2} \mu_{\mathrm{O}_{2}}$, arising from the fact that both alumina and water are in equilibrium, and there is enough of these species to consider them as a reservoir. The equilibrium also imposes specific boundaries to the allowed values of the chemical potential. For $\alpha-\mathrm{Al}_{2} \mathrm{O}_{3}$ they refer to $\mu_{\mathrm{Al}}$ and $\mu_{\mathrm{O}}$. The lower limit of $\mu_{\mathrm{O}}$ is given by $\frac{1}{3} \mu_{\mathrm{Al}_{2} \mathrm{O}_{3}}-2 \mu_{\mathrm{Al}}^{\text {bulk }}<\mu_{\mathrm{O}}$. If the chemical potential of oxygen falls below this limit the alumina is decomposed into metallic aluminum and gaseous oxygen. The upper limit is limited by the presence of the molecular oxygen $\mu_{\mathrm{O}}<\frac{1}{2} \mu_{\mathrm{O}_{2}}$. The same applies to the presence of $\mathrm{H}_{2} \mathrm{O}$ in the system.

When Eq. (3.7) is applied to $\alpha-\mathrm{Al}_{2} \mathrm{O}_{3}$ the chemical potentials of oxygen and hydrogen are the only independent variables. Assuming hydroxylation without oxidation then $N_{\mathrm{O}}=\frac{3}{2} N_{\mathrm{Al}_{2} \mathrm{O}_{3}}+\frac{1}{2} N_{w}$ and the dependence on $\mu_{\mathrm{O}}$ can be eliminated. If hydrogen is not initially present in the system, the equilibrium equation simplifies to

$$
\begin{aligned}
G_{S}= & N\left(G_{\mathrm{Al}_{2} \mathrm{O}_{3}}-T \Delta S_{\mathrm{Al}_{2} \mathrm{O}_{3}}\right) \\
& +N_{\mathrm{H}_{2}}\left(G_{w}-T \Delta S_{w}+k T \ln \left(\frac{p_{w}}{p^{\ominus}}\right)\right) .
\end{aligned}
$$

The general Eq. (3.7) describes the surface at equilibrium with the gaseous species. It has been very successfully applied to $\mathrm{Al}_{2} \mathrm{O}_{3},{ }^{10,22} \mathrm{Fe}_{2} \mathrm{O}_{3},{ }^{21} \mathrm{RuO}_{2},{ }^{23}$ and other oxides. In all cases a clear picture of the surface composition was obtained, but limited to the region of the chemical potential far from the phase boundary. Since the method can be generalized to any system it shows the predictive power of the theoretical approach.

One should notice that Eq. (3.7) is simply a version of the free energy method known also as the Ellingham diagram method. ${ }^{25}$ This approach is dedicated to the description of solid state chemical reactions at equilibrium. In particular the reaction

$$
2 \mathrm{Al}_{2} \mathrm{O}_{3} \rightleftarrows 4 \mathrm{Al}+3 \mathrm{O}_{2}
$$

[related to Eq. (3.2)] can be represented in this way and the equilibrium conditions established constraining the chemical potentials of oxygen, aluminum, and corundum. Also more complicated reactions such as

$$
\begin{aligned}
& 2 \mathrm{Al}(\mathrm{OH})_{3} \rightleftarrows \mathrm{Al}_{2} \mathrm{O}_{3}+3 \mathrm{H}_{2} \mathrm{O}, \\
& 2 \mathrm{AlOOH} \rightleftarrows \mathrm{Al}_{2} \mathrm{O}_{3}+\mathrm{H}_{2} \mathrm{O},
\end{aligned}
$$

can be described in this way. The method becomes very useful in comparing the relative stability of different phases, even allowing different stoichiometry. In such a case each reaction is represented as a sum of elementary reactions (including $\mathrm{H}_{2} \mathrm{O} \rightleftarrows \mathrm{H}_{2}+\frac{1}{2} \mathrm{O}_{2}$ ). By finding the equilibrium conditions for each phase (reaction), the relative stability between different phases can be determined. This requires knowledge of the ground state energies only.

Phase diagrams (also called predominance diagrams) can be constructed and the stability of different phases compared 
as a function of the chemical potential of the constituent elements. The chemical potential can be further translated into experimentally accessible parameters such as $(T, p)$. The aforementioned studies of the surface stability follow this approach.

However, this simple formalism does not provide information on the properties related to the surface. Namely, at the surface the adsorption and desorption processes are crucial in establishing the equilibrium and the underlying bulk may correspond even to the different phase.

Surface specific effects: An important point of the approach presented above is the assumption that the properties of the surface are the same as the bulk, which is not always true.

From the thermodynamic point of view the adsorption energy plays the same role for the surface as the heat (enthalpy) of formation for the bulk phase. But, unlike the enthalpy of formation, the adsorption energy, in the most cases, depends on the surface coverage, $\Theta$. This must be included in the proper description of the surface at equilibrium. As there is no clear border between adsorption and formation of a new phase, it is convenient to follow the suggestion ${ }^{26}$ that at the coverage, at which the differential heat of adsorption becomes lower than the enthalpy of bulk formation, a new compound is formed. For $\Theta$ above this limit the Ellingham thermodynamic approach is fully justified irrespective of considering surface or bulk processes.

A second problem is related to the entropy (free energy) of the bulk and the surface. As the vibrational and configurational properties of the two cases are often different, a description of the equilibrium must take this into account. Reuter and Scheffler ${ }^{23}$ considered the change of the vibrational free energy between the bulk and the (110) surface of $\mathrm{RuO}_{2}$ and have shown that the difference is very small. However, their approach only applies to the stoichiometric surface, where a softening of the vibrational modes is usually observed. For nonstoichiometric surfaces the vibrational free energy is different, especially for strongly adsorbed species. For example, atomic or molecular adsorption results in fairly localized modes. This can be clearly observed for $\mathrm{H}_{2} \mathrm{O}$ dissociation on the $\alpha-\mathrm{Al}_{2} \mathrm{O}_{3}$ (0001) surface. Resulting $\mathrm{OH}$ groups exhibit a precisely defined high frequency mode ${ }^{6}$ around $3800 \mathrm{~cm}^{-1}$, related to the $\mathrm{O}-\mathrm{H}$ stretching vibration. This particular mode is a well established subject of infrared (IR) spectroscopy, and possesses a frequency much higher than the overall phonon spectrum of the substrate. In general, vibrations of any chemisorbed species are stiffer than the bulk. The effect is spatially limited only to the atoms close to the surface.

The sharp distinction of the surface and bulk regions suggest that the free energy, $G_{S}$, of the system may be split into $G_{\text {bulk }}$, related to the underlying substrate and $G_{\text {surf }}$, related to the surface

$$
G_{S}=G_{\text {bulk }}+G_{\text {surf }} .
$$

Each part of the free energy can be further divided into electrostatic and vibrational/configurational contributions: $G_{\text {bulk }}$ $=E_{\mathrm{Al}_{2} \mathrm{O}_{3}}+F_{\text {vib }}$ and $G_{\text {surf }}=E_{\text {surf }}+F_{\text {vib }}$. From this point of view all effects which are specific to the surface become more transparent. In real systems one might expect a rather smooth change of the free energy between the bulk and the surface. ${ }^{20}$ For the purpose of analysis the transition region between the surface and bulk is neglected. There are a variety of methods to calculate the vibrational free energy $F_{\text {vibr }}$ of solids. We refer to the quasiharmonic approximation, where the free energy of the crystal is expressed as

$$
F=k_{B} T \int_{0}^{\infty} d \omega g(\omega) \ln \left(2 \sinh \left(\frac{\hbar \omega}{2 k_{B} T}\right)\right),
$$

where $g(\omega)$ is the phonon density of states. Calculations of this quantity are possible nowadays for the majority of crystalline phases with the accuracy of an ab initio approach. Accurate calculations of the vibrational spectrum of complicated surfaces with high accuracy DFT methods are still problematic though usually rely on molecular dynamics (MD) simulations. The quasiharmonic approximation of free energy and MD simulations will be applied to corundum later in this paper.

The simplest way to include surface effects is to calculate the coverage dependence of the adsorption energy $H_{\text {ads }}(\Theta)$ of water. This can easily be done within the DFT approach, utilizing large supercells. Once the adsorption energy at several discrete coverages is known the differential heat of adsorption can be approximated as $q=E_{0}$ $+\Theta \partial E / \partial \Theta$. The analytical form of $q(\Theta)$ can be established. As the method of calculation gives freedom in the choice of the configuration of the adsorbed atoms at the surface, various adsorption modes can be investigated. For example, a subsurface adsorption, a mixed surface-subsurface adsorption or other adsorption modes can be examined, and the most stable adsorption geometry established with respect to the external conditions. The heat of water adsorption is presented in Sec. V.

A more accurate thermodynamic description of equilibrium at the surface requires further specification of the model. We limit further consideration to surface adsorption only and assume that $M$ adsorption sites are available at the surface. $N$ adsorbed molecules occupy exactly $N \leqslant M$ sites. Once they adsorb in the most stable site they do not diffuse between the sites or interact with each other. These limitations simplify the problem without losing the generality.

The canonical partition function for the adsorbed molecules can be written as ${ }^{27}$

$$
\mathcal{Z}_{\mathrm{ads}}=\frac{M !}{N !(M-N) !} z_{\mathrm{ads}}^{N},
$$

where the first part is related to the configurational distribution of the adsorbed species and $z_{\text {ads }}$ is the vibrational partition function of each adsorbed molecule (atom). $z_{\text {ads }}$ includes all harmonic degrees of freedom of the adsorbate. Thus for localized adsorption there are 3 degrees $(x, y, z)$ per adsorbed atom if $k T \ll E_{\text {ads }}$. The harmonic partition function for the adsorbed atom reads

$$
z_{\mathrm{ads}}=\sum_{i}^{n} \exp \left(-\epsilon_{i} / k T\right)[1-\exp (-h \omega / k T)]
$$



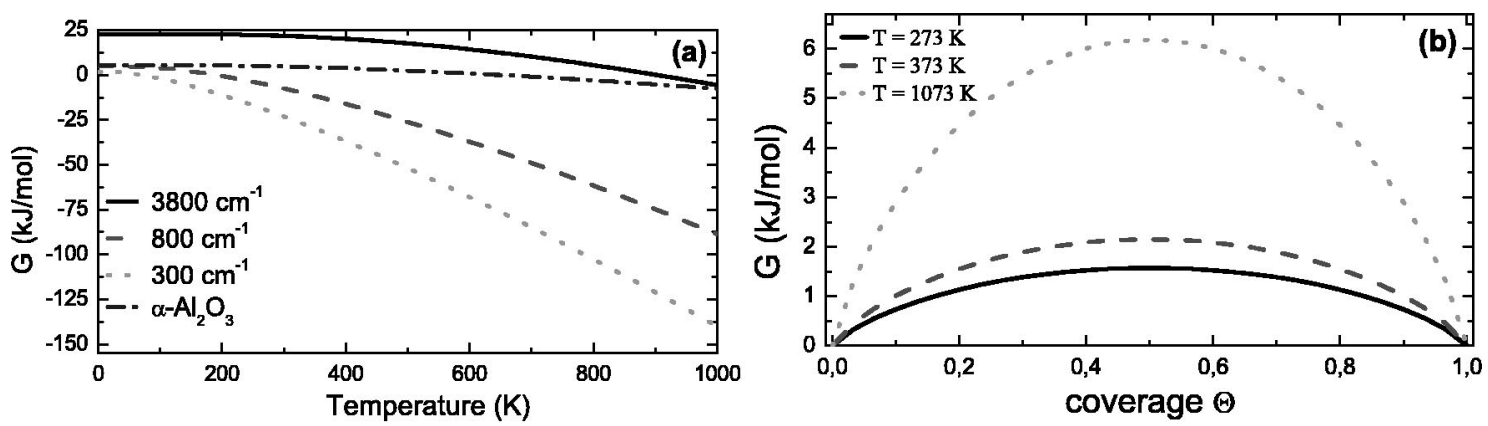

FIG. 3. (a) The vibrational part of the surface free energy compared to the free energy of phonons in the bulk. $F_{\text {vib }}$ is presented per one degree of freedom. Dot-dashed line is the bulk substrate, solid for the contribution from $\mathrm{OH}$ vibration $\left(3800 \mathrm{~cm}^{-1}\right)$, dashed from the vibration of adsorbed oxygen $\left(800 \mathrm{~cm}^{-1}\right)$ and dotted results from the low frequency localized mode at $\left(300 \mathrm{~cm}^{-1}\right)$. (b) The configurational part of the free energy comes from the entropy of particles adsorbed at the surface. Solid, dashed, and dotted lines are for temperatures of 273,373 , and $1073 \mathrm{~K}$, respectively.

and $\epsilon_{i}=\left(h \omega_{i} / 2\right)$, where $\omega_{i}$ is the harmonic frequency of the degree of freedom and the summation runs over all degrees of freedom per adsorption site.

The Helmholtz free energy related to the adsorbed species can be written as

$$
\begin{aligned}
F_{\mathrm{ads}}= & E_{\mathrm{ads}}+F_{\mathrm{vib}}+F_{\mathrm{conf}} \\
= & E_{\mathrm{ads}}-3 N k T \ln \left(\Pi z_{a}\right)-k T(M \ln (M /(M-N)) \\
& +N \ln ((M-N) / N)) .
\end{aligned}
$$

The contribution to the free energy coming from the surface is presented in Fig. 3. The surface coverage $\Theta=N / M$ is introduced for the clarity of presentation. The vibrational part of the free energy is important over all temperature ranges [Fig. 3(a)]. At low temperatures the zero point vibrations of the high frequency modes related to the $\mathrm{OH}$ groups dominate over the lattice excitations. The configurational free energy is significantly lower as depicted on Fig. 3(b). Its significance increases with temperature. At coverages of $\Theta$ $=0$ and $\Theta=1$ the configurational part of the free energy equals zero and at this limit reduces the problem to the Ellingham method.

By including the free energy of the adsorbate, Eqs (3.7) and (3.10) can be complemented and the equilibrium becomes

$$
\begin{aligned}
& G_{S}(T, p)=\left(E_{\mathrm{Al}_{2} \mathrm{O}_{3}}+F_{\mathrm{vib}}\right)+G_{\mathrm{gas}}, \\
& G_{S}(T, p)=E_{\mathrm{slab}}+F_{\mathrm{vib}}+F_{\text {ads }},
\end{aligned}
$$

where $G_{\text {gas }}$ represents the free energy of the gas phase of elements present in the system and $E_{\text {slab }}=E_{\mathrm{Al}_{2} \mathrm{O}_{3}}+E_{\text {surf }}$. Assuming now that (a) both clean and adsorbed surfaces are located on a stack of the same bulk material and (b) no vacancies or other modifications are created in the bulk due to the surface adsorption, we can calculate the surface free energy and the equilibrium surface coverage. The surface energy equals $\Delta G\left(0, p^{\ominus}\right) / A=\left(E_{\mathrm{Al}_{2} \mathrm{O}_{3}}-E_{\text {slab }}\right) / A$ at $T=0 \mathrm{~K}$. $E_{x}$ is the energy calculated by the DFT method and A is the surface area in the supercell. Taking into account (a) and (b) $F_{\text {vib }}$ is the same for both sides of the equilibrium equation. So the vibrational properties of the bulk can be omitted.

The ground state electronic energy determines the phase stability far from the phase boundary, while entropic contri- butions become more important in close proximity to the two different surface phases (coverages). As shown by Wang et al. ${ }^{10}$ close to the standard thermodynamic conditions and stoichiometric composition of the surface (with respect to $\mathrm{Al}_{2} \mathrm{O}_{3}$ and $\mathrm{H}_{2} \mathrm{O}$ ) the surface energy does not depend on the chemical potential of oxygen, and is determined only by the electronic energies in the ground state. This thermodynamic region is the most interesting and we focus in the following on the properties of the hydroxylated $\alpha-\mathrm{Al}_{2} \mathrm{O}_{3}$ (0001) surface at close to $p^{\ominus}$.

Knowing $F_{\text {ads }}$ [Eq. (3.13)] provides the possibility of determining the surface composition at equilibrium. The equity of the chemical potential of the water in the vapor and adsorbed form leads to the the equation of state: $\Phi / k T$ $=-\ln (1-\Theta)$ and $\mu / k T=-\ln [\Theta /(1-\Theta) z]$, where the surface coverage is introduced and $\Phi$ is the surface equivalent of pressure (according to $d E=T d S-\Phi d M+\mu d N, d M$ is the variation of the number of adsorption sites). As the adsorbed phase is in equilibrium with the surrounding gas we have the chemical potential of vapor $\mu=\mu^{0}(T)+\ln \left(p / p^{\ominus}\right)$ and the adsorption isotherm reads:

$$
\Theta(T, p)=\frac{z(T) \exp \left(\mu^{0}(T) / k T\right) \frac{p}{p^{\ominus}}}{1+z(T) \exp \left(\mu^{0}(T) / k T\right) \frac{p}{p^{\ominus}}} .
$$

This is the well-known Langmuir adsorption isotherm. It gives the amount of the adsorbed gas at given $(T, p)$ at equilibrium.

The model can be easily extended, as more molecules can be adsorbed over each site. If the first adsorbed layer becomes a substrate for another layer and so on, this leads to the BET model for adsorption. ${ }^{25}$ To estimate the equilibrium in such a system the adsorption energy and the partition function of each layer must be known. Both quantities can be calculated, the calculations of the exact partition function $Z$, become very laborious for more than a monolayer of water, due to configurational complexity which is coupled to the dynamical part of $Z$. We present an alternative, equivalent formulation of the above approach instead, which is simpler from a computational point of view, as it permits appropriate averaging without loss of accuracy. 


\section{STATISTICAL MODEL OF SURFACE EQUILIBRIUM}

We assume that chemisorption, dissociation and physisorption on the (0001) $\alpha-\mathrm{Al}_{2} \mathrm{O}_{3}$ surface can be described by an extension of the BET model. ${ }^{25}$ Transformation between corundum and aluminum hydroxide is given by $\mathrm{Al}_{2} \mathrm{O}_{3}$ $+3 \mathrm{H}_{2} \mathrm{O} \rightleftharpoons 2 \mathrm{Al}(\mathrm{OH})_{3}$. Thus oxide sites can be described as an inert column of solid capped by $3 \mathrm{O}$ atoms, while the hydroxylated surface can be described as a column capped by $6 \mathrm{OH}$ groups. The mechanism is

$$
\begin{array}{ll}
A_{0}+3 W \rightleftharpoons B_{0}, & \\
A_{n}+W \rightleftharpoons A_{n+1} & \text { for } n \geqslant 1, \\
B_{n}+W \rightleftharpoons B_{n+1} & \text { for } n \geqslant 1,
\end{array}
$$

where $W$ is water, $A_{n}$ is an oxide site $\left(\alpha-\mathrm{Al}_{2} \mathrm{O}_{3}\right)$ covered by $n$ water molecules, and $B_{n}$ is a hydroxide site $\left(\mathrm{Al}(\mathrm{OH})_{3}\right)$ covered by $n$ water molecules. Water is assumed to be physisorbed for $n \geqslant 2$ on $A$ and $n \geqslant 1$ on $B$. The coverage is normalized as $\sum_{n=0}^{\infty} \theta_{A_{n}}+\sum_{n=0}^{\infty} \theta_{B_{n}}=1$. The number of physisorbed molecules on an $A$ site is $\sigma_{A}=\sum_{n=2}^{\infty} n_{i} \theta_{A_{i}}$ and may be interpreted as a coverage. However, it has different mathematical properties from the coverage, $\theta$. The equilibrium equations are

$$
\begin{aligned}
& K_{0} \theta_{A}\left(\frac{p_{w}}{p^{\ominus}}\right)^{3}=\theta_{B}, \\
& K_{1 A} \theta_{A}\left(\frac{p_{w}}{p^{\ominus}}\right)=\theta_{A_{1}}, \\
& K_{P} \theta_{A_{n}}\left(\frac{p_{w}}{p^{\ominus}}\right)=\theta_{A_{n+1}} \quad \text { for } n \geqslant 1, \\
& K_{P} \theta_{B_{n}}\left(\frac{p_{w}}{p^{\ominus}}\right)=\theta_{B_{n+1}} \text { for } n \geqslant 0 .
\end{aligned}
$$

For $p_{w}<K_{P}^{-1} p^{\ominus}$, the solution is

$$
\begin{aligned}
& \theta_{A_{0}}=\frac{1}{1+K_{1 A}\left(\frac{p_{w}}{p^{\ominus}}\right)+K_{0}\left(\frac{p_{w}}{p^{\ominus}}\right)^{2}+\frac{K_{0} K_{p}\left(\frac{p_{w}}{p^{\ominus}}\right)^{4}}{1-K_{p}\left(\frac{p_{w}}{p^{\ominus}}\right)}} \\
& \theta_{A_{1}}=K_{1 A}\left(\frac{p_{w}}{p^{\ominus}}\right) \theta_{A}, \\
& \theta_{B_{0}}=K_{0}\left(\frac{p_{w}}{p^{\ominus}}\right)^{3} \theta_{A}, \\
& \sigma_{A}=\frac{K_{1 A} K_{p}\left(\frac{p_{w}}{p^{\ominus}}\right)^{2}}{\left(1-K_{p} \frac{p_{w}}{p^{\ominus}}\right)^{2}} \theta_{A}, \\
& \sigma_{B}=\frac{K_{0} K_{p}\left(\frac{p_{w}}{p^{\ominus}}\right)^{4}}{\left(1-K_{p} \frac{p_{w}}{p^{\ominus}}\right)^{2}} \theta_{A} .
\end{aligned}
$$

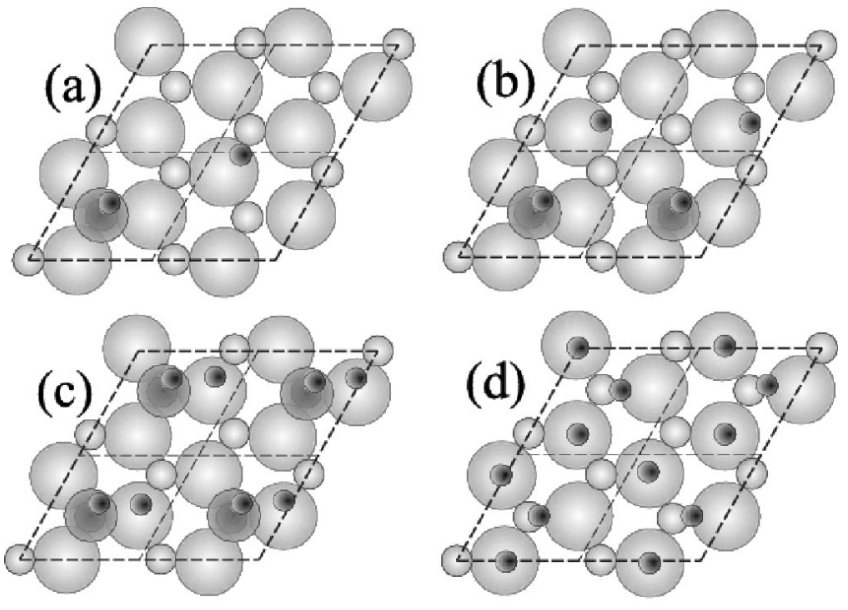

FIG. 4. Surface of corundum at various water coverages considered in the text. (a) $2.6 \mathrm{OH} / \mathrm{nm}^{2}$, (b) $5 \mathrm{OH} / \mathrm{nm}^{2}$, (c) $10.0 \mathrm{OH} / \mathrm{nm}^{2}$, (d) gibbsite-like surface terminated by hydrogen. Large spheres represent oxygen, small gray are for aluminum, and dark gray are hydrogen. The darker large spheres represent adsorbed oxygen.

From this equation it is obvious that the number of adsorbed layers becomes infinite when $p_{w} \rightarrow K_{1 A}^{-1} p^{\ominus}$, which means that $K_{1 A}^{-1} p^{\ominus}$ must be the vapor pressure, $p_{v}$, of water at the adsorption temperature. This provides both a recipe for the calculation of $K_{1 A}$, and an explanation for the restriction $p_{w}<K_{1 A}^{-1} p^{\ominus}$. The vapor pressure, $p_{v}$ at the adsorption temperature, $T$, can be extrapolated $\left[\ln \left(p_{v} / p^{\ominus}\right)=\ln \left(p_{0} / p^{\ominus}\right)\right.$ $\left.+\left(H_{v} / R T_{0}^{2}\right)\left(T-T_{0}\right)\right]$ from the known vapor pressure $p_{0}$ $=113.3 \mathrm{~Pa}$ at the temperature $T_{0}=255.85 \mathrm{~K}$.

The equilibrium constants $K_{0}$ and $K_{1 a}$ are calculated from the properties of $A_{0}, A_{1}, B_{0}$, and $W$ by standard statistical thermodynamic equations. The application of our model to other systems is straightforward. In addition to the calculated energies, we need characteristic vibrational frequencies. They can be calculated directly by an $a b$ initio approach as presented below.

\section{RESULTS}

The coverage dependence of the water adsorption energy was studied using a $2 \times 2$ supercell (see Fig. 1) $\mathrm{H}_{2} \mathrm{O}$ molecules were placed on the relaxed (0001) surface of corundum. The adsorption energy is defined as $E_{\text {ads }}=\left(E_{\text {slab }}\right.$ $\left.-E_{\mathrm{Al}_{2} \mathrm{O}_{3}}-N E_{\mathrm{H}_{2} \mathrm{O}}\right) / N$, where $E_{\text {slab }}$ is the energy of the slab, $E_{\mathrm{Al}_{2} \mathrm{O}_{3}}$ is the energy of the clean relaxed surface, and $E_{\mathrm{H}_{2} \mathrm{O}}$ is the energy of an isolated water molecule in vacuum. The dissociative and molecular adsorption energies were compared for coverages below $1 \mathrm{ML}$.

The size of the supercell allows four different coverages below $1 \mathrm{ML}$. The 1/4 ML is the lowest coverage, modeled by placing one $\mathrm{H}_{2} \mathrm{O}$ molecule at the $(2 \times 2)$ surface. The different adsorption geometries which are possible are depicted in Fig. 1. The most stable configurations are presented in Figs. 4(a) $-4(\mathrm{c})$.

For coverages lower than $1 \mathrm{ML}$, water dissociates on the surface. The hydroxyl group binds to the surface cation at a distance of $1.7 \AA$. For all coverages these ad-OH groups are tilted from their ideal vertical position and the $\mathrm{H}-\mathrm{O}-\mathrm{Al}$ 
angle ranges between $110^{\circ}$ and $123^{\circ}$. The second hydrogen from the dissociated water binds to oxygen at the surface and the most stable configurations always result from the largest spacing between the ad- $\mathrm{OH}$ and surface $\mathrm{OH}$ groups as observed by Hass et al. ${ }^{6}$ At a coverage of $1 \mathrm{ML}$ the H-H separation at the surface equals $3.12 \AA$, increasing to $4.90 \AA$ at $1 / 4 \mathrm{ML}$. Also at coverages below $1 \mathrm{ML}$, adsorption of water creates a large surface distortion accompanied by migration of the cations into the subsurface region. At 1 ML every surface cation is already saturated with one $\mathrm{OH}$ group, which gives a total coverage of $10 \mathrm{OH} / \mathrm{nm}^{2}$. This surface is referred to as fully hydroxylated. When more water is added to this surface, it forms an H-bonded overlayer. Up to two additional water overlayers were studied. A very stable configuration exists at a coverage of $2 \mathrm{ML}$, when one water molecule per unit surface area is added. This configuration consists of a plane hexagonal network, where water molecules lie flat at the distance of $2.2 \AA$ above the surface. Hydrogen is pointing toward the surface ad-OH group, while these $\mathrm{OH}$ groups are attached to the oxygen of the $\mathrm{H}_{2} \mathrm{O}$. This gives a very stable hydrogen bonded network. Addition of a second overlayer destroys this stable configuration, which is reflected in a drop in the adsorption energy close to the heat of water vaporization.

The second type of surface used in the present study was the gibbsite-like surface. It was modeled by the same slab as the (0001) $\alpha-\mathrm{Al}_{2} \mathrm{O}_{3}$ with the surface cation replaced by three hydrogen atoms ${ }^{28}$ Formally this is equivalent to the adsorption of three water molecules (donating six hydrogens) and the creation of $\mathrm{Al}_{2} \mathrm{O}_{3}$ (this utilizes two $\mathrm{Al}$ cations removed from the surface). Since two cations are involved, this process requires two surface area units, however the final result gives the $1 \times 1$ structure. The gibbsite-like surface was optimized, which gives two vertical $\mathrm{OH}$ groups and one hydrogen involved in a $\mathrm{H}$ bond per unit surface area, similar to that shown in Ref. 28. For this surface stoichiometry dissociative adsorption is not energetically favorable at any coverage. The first monolayer does not form as stable structure as on the fully hydroxylated surface, since there is an excess of hydrogen preventing the formation of stable bonds. At this coverage $\mathrm{H}_{2} \mathrm{O}$ stands "vertically" pointing one hydrogen atom toward the surface oxygen, while leaving the second hydrogen dangling free. Addition of the second monolayer changes the situation, as a hexagonal overlayer, similar to the one at the fully hydroxylated surface is formed $2.5 \AA$ above the surface. The enhanced stability of this hydrogen bonded network is reflected in an increased adsorption energy.

The calculated heat of adsorption of water on the (0001) surface of $\alpha-\mathrm{Al}_{2} \mathrm{O}_{3}$ and $\mathrm{Al}(\mathrm{OH})_{3}$ is presented in Fig. 5. For the (0001) surface of corundum two distinct regions are observed: below $1 \mathrm{ML}$, where water dissociates and forms a strong bond with the surface, and above $1 \mathrm{ML}$, where a rapid drop of adsorption energy $\mathrm{H}$ reflects weakly physisorbed molecules. The numerical values of the adsorption energies are presented in Table I.

The vibrational properties of adsorbed water were determined via molecular dynamics simulations. Two independent sets of simulations were done, one for the corundum surface, another for the gibbsite-like surface. In each case the opti-

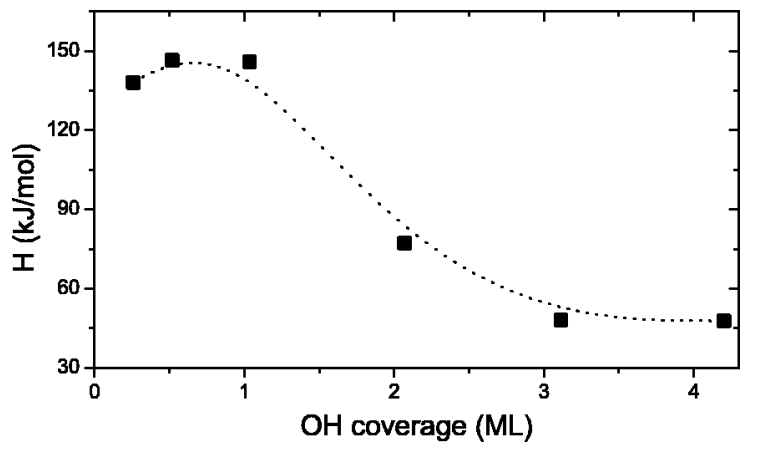

FIG. 5. The differential heat of adsorption of water on the (0001) surface of $\alpha-\mathrm{Al}_{2} \mathrm{O}_{3}$. Dissociative adsorption occurs at coverages from 0 to $10 \mathrm{OH} / \mathrm{nm}^{2}$ ( 0 to $1 \mathrm{ML}$ ); at higher coverages molecular adsorption is preferred. The line is drawn as a guide to the eyes.

mized ground state was used as the starting configuration. For the corundumlike surface, independent simulations were done for the fully hydroxylated surface and the surface with a water overlayer. The same was done for the gibbsite-like surface.

The temperature of the system was fixed to $T=350 \mathrm{~K}$ by a Nose-Hoover thermostat. The mass of hydrogen was replaced by that of deuterium, which gives the possibility of using an integration step of $0.5 \mathrm{fs}$. After each step the electronic structure was reoptimized and the Hellman-Feynman forces utilized to perform the time evolution of the system. The total time of the simulation was 1 ps.

During the simulation runs all structures were stable and no desorption of water was observed. Proton transfer occurs, especially for surfaces with an adsorbed overlayer of water. Proton exchange takes place between the surface $\mathrm{OH}$ groups and adsorbed water, indicating a rather dynamic hydrogen bonding structure.

The characteristic frequencies of vibration were determined by the center of a Gaussian fitted to the power spectrum. As anharmonic effects and mode coupling already play an important role at the temperature of the simulations, the frequencies have to be considered as the average.

The most important surface modes are presented in Fig. 6 and compared to the phonon spectrum of the bulk. ${ }^{29}$ The difference is striking, the $\mathrm{OH}$ stretching mode is located far above any lattice excitations. The second highest frequency belongs to the oxygen-cation stretching mode. The most important frequencies are $720 \mathrm{~cm}^{-1}$ related to the surface cations, and $485 \mathrm{~cm}^{-1}$ for the anions. They are represented by the dotted lines in Fig. 6 and a slight stiffening is seen. The stretching vibration of adsorbed $\mathrm{OH}$ is approximately $1050 \mathrm{~cm}^{-1}$ for the $\mathrm{Al}-\mathrm{O}$ bond. The principal $\mathrm{O}-\mathrm{H}$ stretching

TABLE I. The water adsorption energies (in $\mathrm{kJ} / \mathrm{mol}$ ) on the stoichiometric and hydroxylated $\left[\mathrm{Al}(\mathrm{OH})_{3}\right]$ surfaces of (0001) $\alpha$-alumina. Dissociative adsorption is marked with $(*)$ and it is shown when preferred to molecular adsorption.

\begin{tabular}{ccccccc}
\hline \hline Surface & $\frac{1}{4}$ & $\frac{1}{2}$ & $\frac{3}{4}$ & 1 & 2 & 3 \\
\hline $\mathrm{Al}_{2} \mathrm{O}_{3}$ & $133.1^{*}$ & $146.4^{*}$ & $150.5^{*}$ & $146.3^{*}$ & 75.3 & 47.7 \\
$\mathrm{Al}(\mathrm{OH})_{3}$ & & & & 56.9 & 60.3 & 51.1 \\
\hline \hline
\end{tabular}




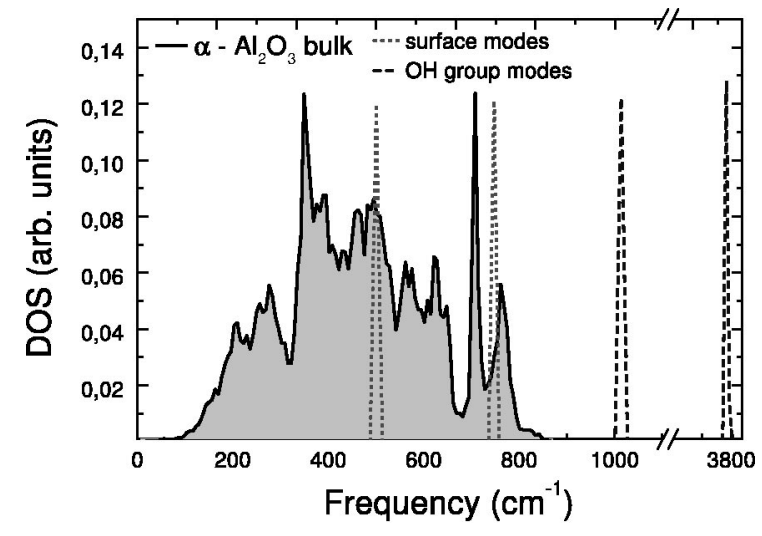

FIG. 6. The comparison of the vibrational density of states for the bulk $\alpha-\mathrm{Al}_{2} \mathrm{O}_{3}$ and the localized modes related to adsorbed water. The solid black line and shaded area represent the bulk properties, the dotted line is related to the vibrational frequencies of surface aluminum and oxygen. Dashed lines represent the frequency region of the hydroxyl group vibration.

frequency is observed at $3780 \mathrm{~cm}^{-1}$ for the topmost hydroxyl group. The different nature of the hydroxyl group at the surface gives further splitting of this band. The formation of the hydrogen bond is indicated by the frequency of $3300 \mathrm{~cm}^{-1}$.

The surface modes important to our model for the surface of corundum $\left(A_{0}\right)$ are the vibrations of the surface anions consisting of six frustrated horizontal translations $(6 \times)$, at $700 \mathrm{~cm}^{-1}$ and the frustrated vertical translations $(3 \times)$, at $600 \mathrm{~cm}^{-1}$. For the gibbsite-like surface $\left(B_{0}\right)$ : frustrated vertical translation $(6 \times)$, at $400 \mathrm{~cm}^{-1} ; \mathrm{OH}$ stretch $(6 \times)$ at $3780 \mathrm{~cm}^{-1}$; frustrated horizontal translations $(12 \times)$, at $550 \mathrm{~cm}^{-1}$; frustrated rotation $(12 \times), 400 \mathrm{~cm}^{-1}$. For chemisorbed water, frustrated translation $(3 \times)$, at $200 \mathrm{~cm}^{-1}$; frustrated rotation $(3 \times)$, at $685 \mathrm{~cm}^{-1}$; vibrations $(3 \times), 3490 \mathrm{~cm}^{-1}$. For water we assume three translations defined by the molar mass, $0.01802 \mathrm{~kg} / \mathrm{mol}$, three rotations, $27.88,14.52,9.27 \mathrm{~cm}^{-1}$, three vibrations, $3657.1,1594.8$, and $3755.8 \mathrm{~cm}^{-1}$. In all cases for simplicity we have taken the average frequency of the $\mathrm{OH}$ stretching for adsorbed water. Other modes belong to the bulk and they do not change during any surface processes.

Combining all these results, the equilibrium surface coverage of $\mathrm{OH}$ as a function of temperature and pressure can be calculated. The results are summarized in Fig. 7. At low temperatures the hydroxylated surface is favored and this surface is stable even at UHV conditions and room temperature. Dehydroxylation is very rapid as the temperature rises, especially at low pressures.

The boundary between the gray and black regions in Fig. 7 divides the phase space into two different surface compositions: in the black region the $\mathrm{Al}(\mathrm{OH})_{3}$-like surface is the most stable, while in the gray region water is dissociated on the $\mathrm{Al}_{2} \mathrm{O}_{3}$ surface.

In Fig. 8 the cross sections of Fig. 7 along $p=p^{\ominus}$ are presented. At atmospheric pressure the surface dehydroxylation starts at around $700 \mathrm{~K}$ and the surface becomes free of water at around $900 \mathrm{~K}$. Below $400 \mathrm{~K}$ the gibbsite-like surface appears, but as can be seen it is very rapidly covered by water.

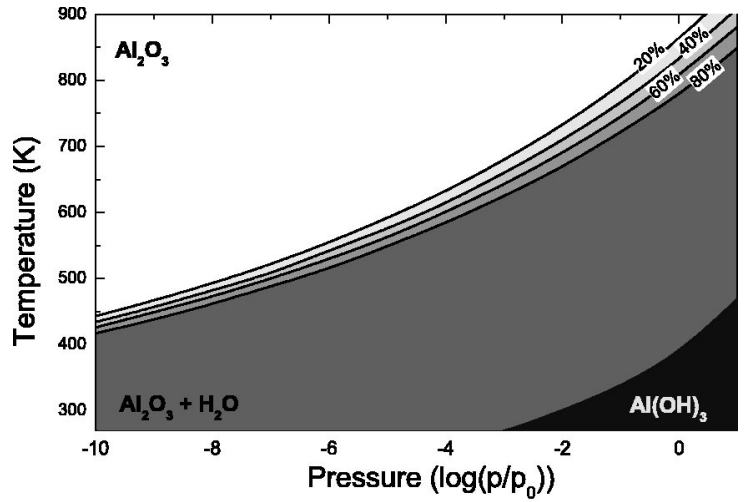

FIG. 7. The phase diagram of the $\alpha-\mathrm{Al}_{2} \mathrm{O}_{3}$ (0001) surface with respect to the pressure of water vapor and temperature. White region is a clean Alterminated surface. The contours are plotted at every 1/5 ML. The black region represents thermodynamic conditions where the $\mathrm{Al}(\mathrm{OH})_{3}$ surface is dominating.

In comparison to the experiment, the fully hydrated surface reported by Eng et al. ${ }^{4}$ can be interpreted by our result, as this type of surface is the most stable, or possesses the lowest free surface energy at room temperature. It was suggested ${ }^{4}$ that an overlayer of water is present on this surface, which is in agreement with our studies (Fig. 8). The single overlayer of water binds to the gibbsite-like surface at a distance of $2.5 \AA$ above the surface oxygen.

Experiments investigating the adsorption ${ }^{13}$ and desorption ${ }^{14}$ of water on single-crystal $\alpha-\mathrm{Al}_{2} \mathrm{O}_{3}$ reveal that the hydroxyl coverage saturates at around $3 \mathrm{OH} / \mathrm{nm}^{2}$ at low water vapor pressures. Such a hydroxylated surface is stable up to $350 \mathrm{~K}$ where rapid $\mathrm{H}_{2} \mathrm{O}$ desorption occurs under UHV. This is the temperature range at which the lowest surface energy of hydrated alumina switches to the dehydrated phase as shown in Fig. 7. Isotopic desorption experiments ${ }^{13}$ showed significant mixing of the oxygen between water and surface oxygen. The process of hydroxylation was recently imaged by $\mathrm{AFM}^{9}$ and revealed the very important role of defects. Our model describes the terrace without defects, which are important centers of hydroxylation. We propose that at low $\mathrm{OH}$ coverages the hydroxylation takes place mainly at surface imperfections and that explains both the

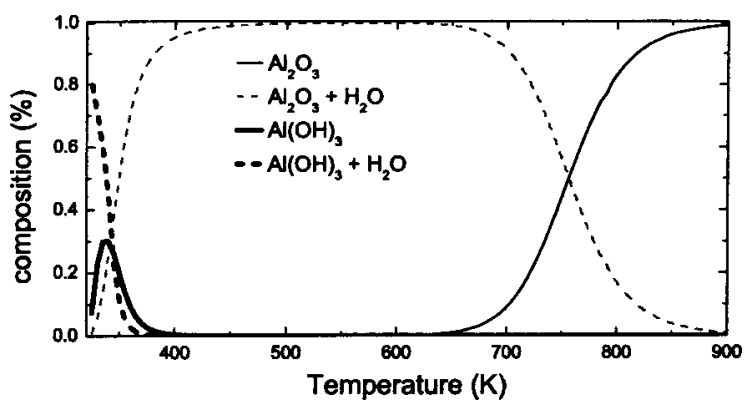

FIG. 8. The cross section of the equilibrium $\mathrm{OH}$ coverage (Fig. 7) of the $\alpha-\mathrm{Al}_{2} \mathrm{O}_{3}(0001)$ surface at $p=1 \mathrm{~atm}$. The thin solid black line represents the clean surface, while the thin dashed line represents the hydroxylated surface. The thick solid line represents the region where $\mathrm{Al}(\mathrm{OH})_{3}$ appears, and the thick dashed line represents the gibbsite-like surface with a water overlayer. 
observed broad range of desorption energies and the isotopic scrambling.

Liu et al $^{30}$ found the water vapor threshold pressure for the total hydroxylation of $\alpha-\mathrm{Al}_{2} \mathrm{O}_{3}$ to be $133 \mathrm{~Pa}$. Our calculations yield a somewhat higher threshold $(250 \mathrm{~Pa})$. The threshold pressure is interpreted as evidence for a crossover from adsorption at defects to adsorption on terraces, while the present study shows that the threshold relates to the change of surface stoichiometry. The agreement between calculated and experimental results could be accidental, as we do not expect such high accuracy, due to well known limitations of DFT methods.

For the systems where the influence of the hydration seem to be of high importance, McHale et al. ${ }^{11}$ show that porous $\alpha$-alumina, obtained from diaspore, is highly hydrated. Heating above $600 \mathrm{~K}$ causes the loss of the surface water accompanied by significant coarsening. The differential heat of adsorption reaches a plateau at around $n$ $=5-10 \mathrm{OH} / \mathrm{nm}^{2}$, which in view of our results could be interpreted as a contribution from the hydroxyl group resulting from the terraces.

Another set of studies investigated the surface in UHV. Ahn and Rablais ${ }^{7}$ annealed the surface under UHV and by TOF-SARS were able to detect randomly distributed hydrogen on the surface, up to $1100{ }^{\circ} \mathrm{C}$. Also both oxygen and aluminum terminations of the surface were found in $\mathrm{UHV}^{8}$ The oxygen termination of the surface has been previously explained in Ref. 10. From our results the partially hydrated surface, terminated by a single aluminum layer is thermodynamically stable. It is thus easy to understand, that both oxygen (bayerite-like) and aluminum terminated surfaces are observed experimentally. Again the role of defects in the stabilization of the $\mathrm{OH}$ groups at the surface cannot be neglected.

To summarize, a method for determination of the surface phase diagram directly from the density functional calculation was presented. As an example of the application we showed that at the low temperature and high pressure of water vapor, the fully hydroxylated, gibbsite-like surface of corundum is the thermodynamically most stable surface. With increasing temperature and/or decreasing water pressure first the hydroxylated (0001) surface of $\alpha$ alumina and later the stoichiometric surface becomes the most stable. The low energy of the hydroxylated surface compares with the experimental observation that the surfaces of alumina are inherently hydroxylated.

\section{ACKNOWLEDGMENTS}

CAMP is sponsored by the Danish National Research Foundation. Allocation of the CPU time by the Danish Center for Scientific Computing within the Grant HDW-1101-05 is kindly acknowledged. The authors would like to thank Dr. J. Davies for reading the paper.

\section{APPENDIX: CHEMICAL POTENTIAL OF GASES AND LIQUIDS}

The temperature and pressure dependence of the chemical potential of gases and liquids is different.
From the microscopic point of view the free energy of the ideal gas can be divided into contributions from translational, rotational, vibrational and electronic degrees of freedom. All these quantities can be calculated and the free energy expressed as a sum: $G=G_{\text {trans }}+G_{\text {rot }}+G_{\text {vib }}+G_{\text {electr }}$. The energy scale related to these degrees of freedom varies over several orders of magnitude ranging from $\sim 10^{-17} \mathrm{eV}$ for translational motion, through $\sim 0.0001 \mathrm{eV}$ for rotational, $\sim 0.1 \mathrm{eV}$ for the vibrational and $\sim 1 \mathrm{eV}$ for the electronic. Only those contributions activated at the temperature of interest $(\sim 0.03 \mathrm{eV})$ are of importance.

For an ideal gases the chemical potential can be expressed as

$$
\begin{aligned}
\mu(T, p) & =\mu\left(T, p^{\ominus}\right)+k_{B} T \ln \left(\frac{p}{p^{\ominus}}\right) \\
& =H\left(T, p^{\ominus}\right)-T S\left(T, p^{\ominus}\right)+k_{B} T \ln \left(\frac{p}{p^{\ominus}}\right)
\end{aligned}
$$

and it is referenced to the standard state. The temperature change of the enthalpy $\Delta H\left(T, p^{\ominus}\right)=H\left(T, p^{\ominus}\right)-H\left(0, p^{\ominus}\right)$ and entropy $\Delta S\left(T, p^{\ominus}\right)=S\left(T, p^{\ominus}\right)-S\left(0, p^{\ominus}\right)$ gives the variation of the chemical potential with $T$,

$\mu(T, p)=\Delta H\left(T, p^{\ominus}\right)+E_{\mathrm{gas}}-T \Delta S\left(T, p^{\ominus}\right)+k_{B} T \ln \left(\frac{p}{p^{\ominus}}\right)$.

In this way, the chemical potential of the gas can be referenced to the ground state energy calculated within the DFT method.

In fact $E_{\text {gas }}$ also contains contributions from the zero point vibrations, which are easy to estimate for simple gases. The quantities $\Delta H\left(T, p^{\ominus}\right)$ and $S\left(T, p^{\ominus}\right)$ can be found in any standard thermodynamic table. ${ }^{31}$

For liquids the situation is more complicated: one takes advantage of the equity of the chemical potential of the saturated vapor and liquid under given thermodynamic conditions. In this case one must go beyond the ideal gas approximation.

${ }^{1}$ H.-J. Freund, Surf. Sci. 500, 271 (2002), and references therein.

${ }^{2} \mathrm{~K}$. Wefers and C. Misra, "Oxides and hydroxides of aluminium," Alcoa Technical Paper No. 19 (Revised), Alcoa Laboratories, 1987.

${ }^{3}$ V. E. Heinrich and P. A. Cox, The Surface Science of Metal Oxides (Cambridge University Press, Cambridge, MA, 1994).

${ }^{4}$ P. J. Eng, T. P. Trainor, G. E. Brown, Jr., G. A. Waychunas, M. Newville, S. R. Sutton, and M. L. Rivers, Science 288, 1029 (2000).

${ }^{5}$ C. Verdozzi, D. R. Jennison, P. A. Schultz, and M. P. Sears, Phys. Rev. Lett. 82, 799 (1999).

${ }^{6}$ K. C. Hass, W. F. Schneider, A. Curioni, and W. Andreoni, Science 282, 265 (1998); J. Phys. Chem. B 104, 5527 (2000).

${ }^{7}$ J. Ahn and J. W. Rabalais, Surf. Sci. 388, 121 (1997).

${ }^{8}$ J. Toofan and P. R. Watson, Surf. Sci. 401, 162 (1998).

${ }^{9}$ C. Barth and M. Reichling, Nature (London) 414, 54 (2001).

${ }^{10}$ X.-G. Wang, A. Chaka, and M. Scheffler, Phys. Rev. Lett. 84, 3650 (2000).

${ }^{11}$ J. M. McHale, A. Auroux, A. J. Perrotta, and A. Navrotsky, Science 277, 788 (1997).

${ }^{12}$ J. M. McHale, A. Navrotsky, and A. J. Perrotta, J. Phys. Chem. B 101, 603 (1997).

${ }^{13}$ J. W. Elam, C. E. Nelson, M. A. Cameron, M. A. Tolbert, and S. M. George, J. Phys. Chem. B 102, 7008 (1998). 
${ }^{14}$ C. E. Nelson, J. W. Elam, M. A. Cameron, M. A. Tolbert, and S. M. George, Surf. Sci. 416, 341 (1998).

${ }^{15}$ C. Wolverton and K. Hass, Phys. Rev. B 63, 024102 (2001).

${ }^{16}$ J. P. Perdew, J. A. Chevary, S. H. Vosko, K. A. Jackson, M. R. Pederson, D. J. Singh, and C. Fiolhais, Phys. Rev. B 46, 6671 (1992).

${ }^{17} \mathrm{http}: / /$ www.fysik.dtu.dk/CAMPOS

${ }^{18}$ W. E. Lee and K. P. D. Lagerlof, J. Electron Microsc. Tech. 2, 247 (1985).

${ }^{19}$ L. P. H. Jeurgens, W. G. Sloof, F. D. Tichelaar, and E. J. Mittemeijer, Phys. Rev. B 62, 4707 (2000).

${ }^{20}$ Z. Lodziana and J. K. Nørskov, J. Chem. Phys. 115, 11261 (2001).

${ }^{21}$ X.-G. Wang, W. Weiss, Sh. K. Shaikhutdinov, M. Ritter, M. Petersen, F. Wagner, R. Schlgl, and M. Scheffler, Phys. Rev. Lett. 81, 1038 (1998).

${ }^{22}$ I. Batyrev, A. Alavi, and M. W. Finnis, Faraday Discuss. 114, 33 (1999).

${ }^{23}$ K. Reuter and M. Scheffler, Phys. Rev. B 65, 035406 (2002).
${ }^{24}$ W. Zhang and J. R. Smith, Phys. Rev. Lett. 85, 3225 (2000); Phys. Rev. B 61, 16883 (2000).

${ }^{25}$ P. W. Atkins, Physical Chemistry (Oxford University Press, Oxford, 1998).

${ }^{26}$ C. I. Carlisle, T. Fujimoto, W. S. Sim, and D. A. King, Surf. Sci. 470, 15 (2000).

${ }^{27}$ T. L. Hill, An Introduction to Statistical Thermodynamics (AddisonWesley, Reading, PA, 1962).

${ }^{28}$ R. Di Felice and J. E. Northrup, Phys. Rev. B 60, R16287 (1999).

${ }^{29}$ Z. Lodziana and K. Parlinski, Phys. Rev. B 67, 174106 (2003).

${ }^{30}$ P. Liu, T. Kendelewicz, G. E. Brown, Jr., E. J. Nelson, and S. A. Chambers, Surf. Sci. 417, 53 (1998).

${ }^{31}$ CRC Handbook of Chemistry and Physics, 64th ed., edited by R. C. Weast (CRC, Boca Raton, FL, 1985). 